\title{
Optimierung des Rekonstruktions-Tools TSNI für genregulatorische Netzwerke mittels Parallelisierung und ein Vergleich mit Time Delay ARACNE
}

\author{
Marco Schäfer, Christian Jetschni, Chong Wang und Heike Pospisil*
}

\section{Zusammenfassung}

Gegenstand dieser Veröfffentlichung ist eine Reimplementierung des TSNI (Time Series Network Identification)Tools zur Rekonstruktion von genregulatorischen Netzwerken aus Zeitserien von Gen-Expressionsprofilen. Das auf gewöhnlichen Differentialgleichungen basierende Tool wurde algorithmisch verbessert und an die Verarbeitung großer Datensätze angepasst. Durch Ausschöpfung des Potentials moderner Multi-CPU und GPU-Architekturen wurden die erforderlichen Berechnungen erheblich beschleunigt. Bei der Überführung des mit Matlab implementierten Tools in die Programmiersprache $\mathrm{C}$ wurde zudem die Bedienbarkeit verbessert.

Durch Einsatz der GPU-beschleunigten Mathematik-Bibliothek MAGMA konnten unter Verwendung eines normalen Desktop-Rechners Leistungssteigerungen um das 18,7-fache bei 11.000 Genen verzeichnet werden. Darüber hinaus rekonstruierte das verbesserte TSNI weitaus mehr biologisch richtige Beziehungen als vergleichbare Anwendungen.
Abstract

The purpose of this work was to optimize a tool for analyzing biological high-throughput data and to generate gene regulatory networks, which are based on ordinary differential equations. For this task the Time Series Network Indentification (TSNI) - tool has been selected. The TSNI-algorithm has been optimized and transferred into the programming language $C$. Computationally intensive sections were parallelized using modern multi-core CPU and GPU architectures as well as functions from the MAGMA-library for the solution and parallelization of more complex mathematical problems. The performance of the algorithm was significantly increased (e.g. the calculation of a network of more than 11.000 genes was 18.7-fold faster with the improved tool compared to the original algorithm). So, large regulatory networks can now be analysed in a moderate time.

Additionally, more true positve network connections were predicted using the adjusted TSNI - tool.

\section{Einleitung}

Biologische Systeme wie Gewebe oder Zellen sind über viele Ebenen (metabolische Netzwerke, Signalwege, genregulatorische Netzwerke) miteinander eng verknüpft und hoch organisiert (Karr et al. 2012). Für ein umfassendes Verständnis dieser Organisation, ist eine ganzheitliche Betrachtung aller Ebenen erforderlich; dies ist Gegenstand der Systembiologie (Reiß 2002), welche derzeit durch die Fortschritte in den Sequenziertechniken zunehmend an Bedeutung gewinnt. Aus diesem Grund und im Zusammenhang mit der sich ebenfalls schnell entwickelnden Computertechnik steht heute weniger die Generierung der Daten, sondern vielmehr die Bewältigung ihrer Auswertung und die Schaffung neuen Wissens im Vordergrund (Jia et al. 2017).

Eine große Herausforderung besteht darin, schnelle und effiziente Algorithmen für die Rekonstruktion von genregulatorischen Netzwerken aus Genexpressionsdaten zu unterschiedlichen Zeitpunkten (Reverse Engineering) zu entwickeln, um Hochdurchsatzdaten in akzeptabler Zeit zu analysieren. Dies ermöglicht eine deutlich schnellere Gene- rierung von neuen Erkenntnissen nicht nur in der Systembiologie, sondern auch in Medizin, Pharmazie und Biotechnologie. Besonders aus medizinischer Sicht ist die Kenntnis molekularer und genetischer Mechanismen wichtig, um Krankheiten mit deregulierten Genen als Ursache besser zu verstehen. Dies kann wiederum neue Angriffsziele für therapeutische Ansätze liefern (Reiß 2002; Jia et al. 2017). Die Kosten der Forschung in den genannten Bereichen können durch das Modellieren von genregulatorischen Netzwerken gesenkt werden, da beispielsweise nicht getestete laborexperimentelle Ansätze 
kostengünstig simuliert und zumindest teilweise ersetzt werden (Reiß 2002; Karlebach \& Shamir 2008).

Ein Software-Werkzeug, das die komplexe Aufgabe der Rekonstruktion von genregulatorischen Netzwerken aus Zeitserien von Genexpressionsdaten realisiert, ist das TSNI (Time Series Network Identification)-Tool (Bansal et al. 2006; Jia et al. 2017). Es modelliert genregulatorische Netzwerke mittels eines Ansatzes, welcher auf Differenzialgleichungssystemen beruht. So wird nicht nur die Topologie eines Netzwerks ermittelt, sondern auch dessen Dynamik (d.h. die durch genregulatorische Beeinflussung hervorgerufenen zeitlichen Konzentrationsveränderungen von Genprodukten) - und dies sogar im zeitkontinuierlichen Raum (Bansal et al. 2006). Damit bildet dieses Tools die biologische Realität deutlich besser ab als beispielsweise Boolesche Netzwerke oder Bayessche Netzwerke.

\section{Methoden und Algorithmen}

\subsection{Algorithmen}

Der Kern von TSNI besteht aus dem Differentialgleichungssystem, welches in Gleichung 1 dargestellt ist. Dieser Ausdruck beschreibt die Rate $\dot{x}_{l}$ der Synthese eines Gens zu einem Zeitpunkt $t_{k}$ in Abhängigkeit von der Konzentration aller anderen Transkripte einer Zelle bzw. des Systems. Die Rate eines Gens hängt von der Art des Einflusses (inhibierend, verstärkend, oder keiner) jeden Gens j $(1 \leq \mathrm{j} \leq \mathrm{N}$, wobei $\mathrm{N}$ die Anzahl der Gene bezeichnet) und dessen Konzentration $x$, zum Zeitpunkt $t$ ab. Hinzu kommt der Einfluss $b_{i l}$ der Perturbationen P. Die Variable $u_{l}$ repräsentiert die I-te Perturbation zu einem Zeitpunkt $t_{k}$ (Bansal et al. 2006).

$\dot{x}_{l}\left(t_{k}\right)=\sum_{j=1}^{N} a_{i j} \cdot x_{j}\left(t_{k}\right)+\sum_{l=1}^{P} b_{i l} \cdot u_{l}\left(t_{k}\right)$

Gleichung 1 lässt sich mit Hilfe der Matrizenschreibweise wie in Formel 2 kompakter darstellen. Dabei steht M für die Anzahl der Zeitpunkte (Bansal et al. 2006).

$$
\dot{X}\left(t_{k}\right)=A \cdot X\left(t_{k}\right)+B \cdot U\left(t_{k}\right) \quad k=1 \ldots M
$$

Anschließend wird der Ausdruck vom zeitkontinuierlichen Raum in den diskreten überführt (vgl. Gleichung 3). Die Ausgangsdaten zur Lösung des Gleichungssystem sind dabei ebenfalls diskret (Bansal et al. 2006).

$$
\dot{X}\left(t_{k+1}\right)=A_{d} \cdot X\left(t_{k}\right)+B_{d} \cdot U\left(t_{k}\right)
$$

Da das Gleichungssystem nur für den Fall $M \geq N+P$ gelöst werden kann, wird zum einen mittels Hauptkomponentenanalyse (engl. Principle Component Analysis; PCA) eine Dimensionsreduzierung von $\mathrm{N}$ und $\mathrm{P}$ durchgeführt. Zum anderen erfolgt mittels kubischer Spline-Interpolation eine künstliche Vervielfältigung der Zeitpunkte $M$. Nach der PCA wird das Gleichungssystem durch Bildung einer Pseudoinversen gelöst und abschließend das Ergebnis mittels der bilinearen Transformation wieder in den zeitkontinuierlichen Raum überführt (Bansal et al. 2006).

\subsection{Methoden}

Für eine effektivere Berechnung wurde der ursprünglich für Matlab implemtierte Algorithmus in die performante und hardwarenähere Programmiersprache C überführt. Zur weiteren Steigerung der Berechnungsgeschwindigkeit kam parallele Programmierung zum Einsatz; dafür wurde die Schnittstelle OpenMP (Dagum \& Menon 1998) verwendet. OpenMP stellt die nötigen Funktionen der Parallelisierung als Erweiterung von $C$ bereit. Dies umfasst das Zerlegen von Problemen in Teilprobleme, die Synchronisation von Threads sowie die Deklaration von gemeinsamen und verteiltem Speicher. Mit Hilfe von OpenMP konnten die Routinen für kleinere Matrix-Multiplikationen parallelisiert werden.

Größere Operationen wurden mittels der MAGMA-(Matrix Algebra on GPU and Multicore Architectures) Bibliothek auf der Grafikkarte (engl. Graphics Processing Unit; GPU) gelöst (Dongarra et al. 2014). Sie basiert auf der CUDA'Schnittstelle, die die Verwendung einer GPU über ihren ursprünglichen Verwendungszweck hinaus ermöglicht und somit die GPU mit ihren hunderten bis tausenden Kernen für massive Parallelisierung bereitstellt (Rauber \& Rünger 2012). Zusätzlich greift MAGMA auf die Pakete LAPACK (Linear Algebra PACKage) und OpenBLAS (Basic Linear Algebra Subprograms) zurück (Dongarra et al. 2014). Die hybriden Algorithmen der MAGMA-Bibliothek nutzen durch intelligente Zerlegung von Problemen in Tasks variierender Granularität den Leistungsumfang von modernen Multi-CPU und GPU-Architekturen aus (Rauber \& Rünger 2012).

\subsection{Daten}

Die für die Weiterentwicklung und Testung des Analyse-Tools genutzten Daten stammen von dem gut erforschten Modellorganismus Arabidopsis thaliana. Im Detail sind es Daten einer Studie zum Übergang vom vegetativem Wachstum zur Blütenbildung. Dabei wurden die Genexpressionsprofile während der Entwicklung von Arabidopsis thaliana in einem Zeitraum von 7 bis 16 Tagen nach der Keimung täglich aufgenommen (Klepikova et al. 2015), so dass die Expressionsdaten von 10 Zeitpunkten im Abstand von einem Tag vorlagen. Um die Vorhersagequalität der biologischen Zusammenhänge abschätzen zu können, wird die Anwendung TD ARACNE (Time Delay Algorithm for the Reconstruction of Accurate Cellular Networks) (Zoppoli et al. 2010) vergleichend herangezogen. TD ARACNE ist ein etabliertes und weit genutztes Tool zur Rekonstruktion von genregulatorischen Netzwerken aus Zeitserien von Genexpressionsprofilen; es verwendet allerdings das Konzept der Transinformation (engl. Mutal Information).

Für die Abschätzung der Vorhersagequalität der gefundenen Interaktionen wurde mit einem Datensatz bereits beschriebener Proteininteraktionen der Datenbank ATPIN ("Arabidopsis thaliana protein interaction network", https://atpin.bioinfoguy.net/cgi-bin/ atpin.pl) verglichen. Release 9 enthält 96.276 Proteininteraktionen. 


\subsection{Intrapolation}

Da die Zahl der untersuchten Gene deutlich die Zahl der Messzeitpunkte übersteigt, ist die Zahl der Freiheitsgrade, die sich aus der Differenz zwischen Genen (N) und Messzeitpunkten (M) ergibt, zu hoch, um verlässliche Aussagen treffen zu können (vgl. Abschnitt 2.1). Aus diesem Grund wird die Zahl der Messzeitpunkte mittels Intrapolation künstlich vergrößert. Eine äquidistante Intrapolation teilt den Zeitbereich zwischen zwei Messpunkten gleichmäßig auf. Liegen jedoch Messwerte zu Zeitpunkten vor, die nicht paarweise jeweils denselben Abstand zueinander aufweisen, muss eine nicht äquidistante Intrapolation durchgeführt werden.

\section{Ergebnisse}

Der ursprüngliche Algorithmus wurde bezüglich des Rechenaufwands deutlich optimiert. Dabei wurde OpenMP zur Parallelisierung von Routinen für kleinere Matrix-Matrix-Operationen genutzt, bei denen der Kopiervorgang auf die GPU keinen Zeitvorteil bringt. Bei der Bewältigung der Parallelisierung mehrerer rechenintensiverer $\mathrm{Ab}$ schnitte wurde die MAGMA-Bibliothek zur Matrix-Matrix-Multiplikation, für die Bildung großer Inversen und zur Singulärwertzerlegung (engl. Singular Value Decomposition; SVD) eingesetzt, wobei letztere den Kern der PCA und der Bildung der Pseudoinversen darstellt.

\subsection{Verbesserung der \\ Algorithmen-Performance}

Das Ergebnis der Reimplementierung des TSNI-Algorithmus in C ist in Abb. 1 dargestellt. Wie zu erkennen ist wird mit steigender Datenmenge der Gewinn an Berechnungszeit deutlich vergrößert: Bei 7.000 Genen ist das verbesserte TSNI 14,4-mal schneller als der ursprüngliche Algorithmus; bei 11.000 Genen konnte bereits ein 18,7-facher Gewinn an Rechenzeit ermittelt werden. Die Grafik zeigt die Zeit für bis zu 11.000 Gene, da Matlab bei Datensätzen >12.000 abbricht.

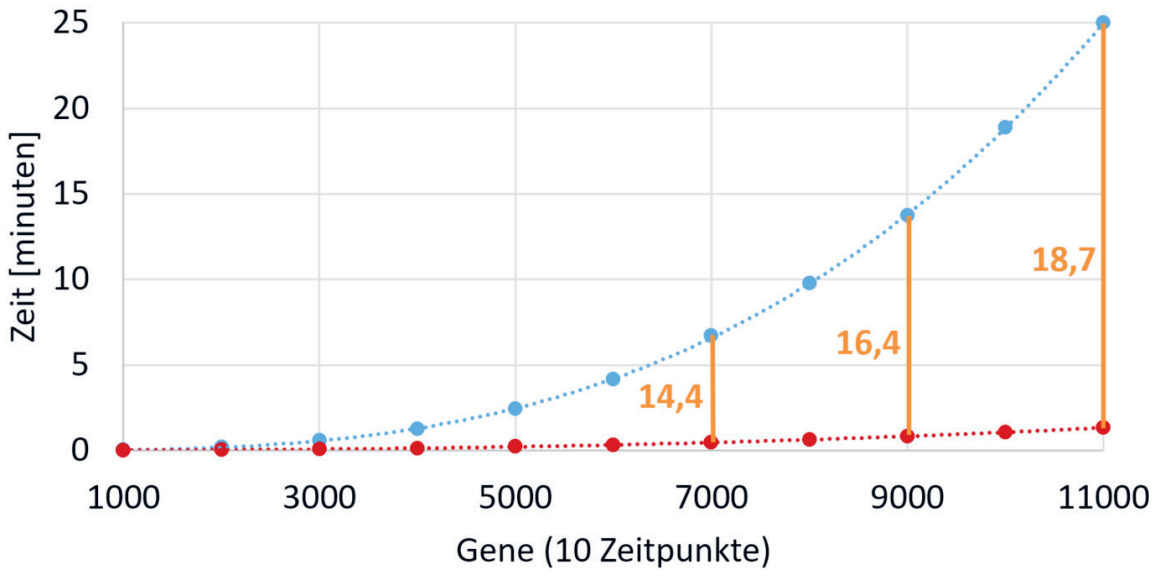

Abb. 1) Berechnungsdauer in Minuten gegen Zahl der Gene. In blau ist die Rechenzeit des ursprünglichen MatlabProgramms und in rot die des optimierten TSNI-Programms dargestellt. In orange sind die Vielfachen der Leistungszuwächse eingezeichnet. [Testsystem: CPU Xeon 1231 V3 (8x3.7 GHz); GPU GM204-GTX 970 (4 GB, 1664x1.2 GHz); $\operatorname{RAM}(16 \mathrm{~GB}, 1.6 \mathrm{GHz})]$

\subsection{Qualität der biologischen Vorhersage}

Beim Vergleich der biologischen Korrektheit der gefundenen Beziehungen zwischen Genen lässt sich erkennen, weniger Genen kaum Unterschiede zwischen den Ergebnissen mit dem hier vorgestellten Ansatz und dem Referenztool TD ARACNE gibt (vgl. Abb. 2).

Enthält der Datensatz jedoch 1.600 Gene oder mehr, werden deutlich mehr korrekte Beziehungen mit Hilfe des auf Differenzialgleichungssystemen basierenden Ansatzes von TSNI gefunden. Dabei konnte eine bis zu 79-fach höhere Anzahl an korrekt vorhergesagten Beziehungen beobachtet werden. dass es bei Datensätzen mit 800 oder

\section{Diskussion}

Das Tool TSNI (Time Series Network Identification) konnte erfolgreich an die Bearbeitung von großen Datensätzen mit über 11.000 Genen angepasst werden. Gezeigt ist die Auswertung für 12.800 Gene, um einen Vergleich mit dem klassischen TSNI-Tool, welches in der Zahl der zu verarbeitenden Gene begrenzt ist, zu ermöglichen. Herkömmliche Tools, wie z.B. TD ARACNE und das ursprüngliche TSNI, sind jedoch nicht in der Lage, solche großen Datenmengen für höhere Organismen (Pflanzen, Tieren und Menschen) mit 20.000 bis 40.000 Genen zu verarbeiten. Um neue Erkenntnisse für die Medizin, Biotechnologie oder Systembiologie zu erlangen, ist es erforderlich, die genregulatorischen Netzwerke, die aus mehreren zehntausend Genen

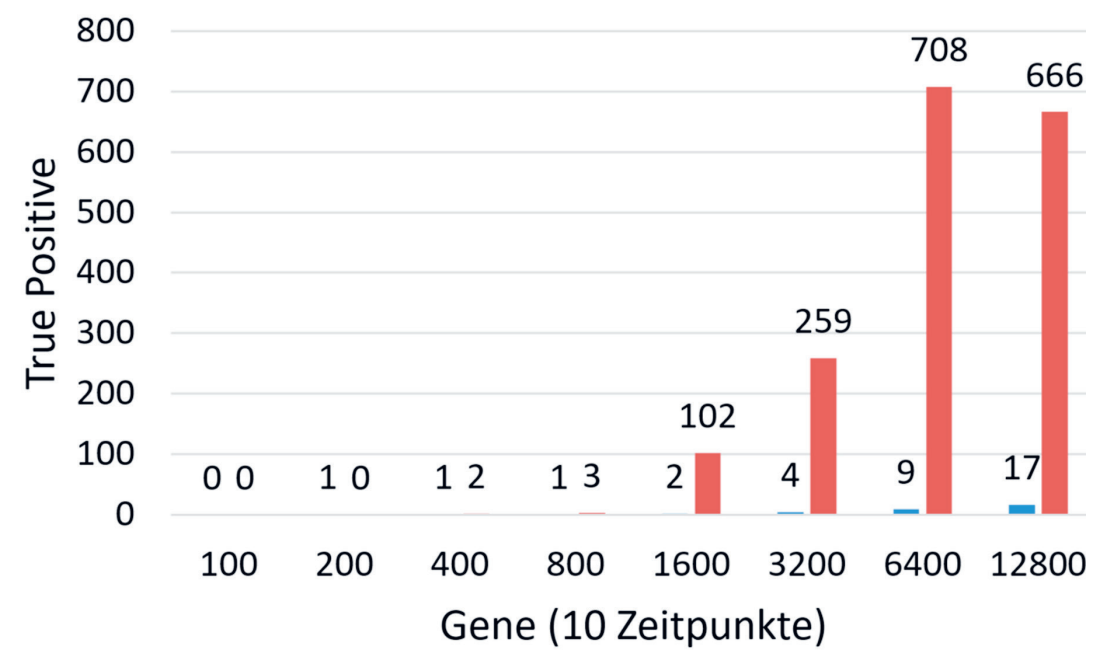

Abb. 2) Anzahl der biologisch tatsächlich korrekten (True Positive) Beziehungen zwischen Genen gegen die Anzahl der Gene, die für die Rekonstruktion genutzt wurden. Dabei ist in blau TD ARACNE und in rot TSNI dargestellt. [TSNI $(P C A=2$, tol=0.2, threshold=0.05); TD ARACNE (tol=0.2, threshold $=0.05)$ ]. 
bestehen, untersuchen zu können, was mit dem hier vorgestellten Ansatz nun möglich ist. Auch eine Analyse des kompletten Genoms von Arabidopsis thaliana (mit ca. 42.000 Genen) ist mit der neu entwickelten Software möglich. Weiterhin wurde die Intrapolation dahingehend verbessert, dass nun auch nicht äquidistante Zeitpunkte berücksichtigt werden können. Dies bedeutet, dass zwischen zwei Zeitpunkten mehr Zwischenpunkte intrapoliert werden, wenn der Abstand dieser zwei Zeitpunkte, im Vergleich zu den übrigen Zeitabständen eines Datensatzes größer ist.

Darüber hinaus ist die Qualität der biologischen Vorhersage der genregulatorischen Netzwerke bei TSNI erheblich besser. Jedoch waren mit dem hier vorgestellten Ansatz viele der Interaktionen der ATPIN-Datenbank nicht ermittelbar, da jeweils nur ein Ausschnitt des Gesamtnetzwerks betrachtet wurde. Desweiteren sind viele der beschriebenen Interaktionen nicht in jedem Zustand der Organismen vorhanden; es kommt beispielsweise nach Störungen oder in verschieden Zellzyklusstadien, unter verschiedenen klimatischen Bedingungen, bei Befall mit Krankheitserregern etc. zu speziellen funktionellen Wechselwirkungen, die nicht in einem einzigen Experiment beobachtet werden können. Die Verbesserung durch das beschriebene Software-Werkzeug wird anhand der erhöhten Zahl an Interaktionen im Vergleich zu denen, die das Tools ARACNE liefert, deutlich.

Die Verbesserungen des Algorithmus und die Parallelisierung haben erhebliche Leistungsverbesserungen ergeben, und die Benutzerfreundlichkeit wurde erhöht. Es bedarf allerdings noch einiger weiterführender Optimierungen, da die Anzahl der korrekten Netzwerkverbindungen noch zu gering ist, was jedoch an der Qualität der verwendeten Daten liegen kann. Wegen der beschriebenen Effizienzsteigerung kann nun aber die Berechnung mit weiteren Datensätzen ohne Einschränkungen realisiert werden, so dass eine weitere Verbesserung der Vorhersagequalität in den nächsten Schritten in Angriff genommen werden kann. Der hier vorgestellte Ansatz unter Nutzung von Hochdurchsatzdaten stellt einen weiteren wichtigen Schritt zum ganzheitlichen Verständnis biologischer Systeme dar.

\section{LITERATUR}

Bansal M, Della Gatta G, Di Bernardo D (2006) Inference of gene regulatory networks and compound mode of action from time course gene expression profiles. Bioinformatics 22(7):815-822. doi: 10.1093/bioinformatics/btl003

Dagum L, Menon R (1998) OpenMP: an industry standard API for shared-memory programming. Computational Science \& Engineering, IEEE 5(1):46-55

Dongarra J, Gates M, Haidar A, Kurzak J, Luszczek P, Tomov S, Yamazaki I (2014) Accelerating Numerical Dense Linear Algebra Calculations with GPUs.

Numerical Computations with GPUs:1-26

Jia B, Xu S, Xiao G, Lamba V, Liang F (2017) Learning gene regulatory networks from next generation sequencing data. Biometrics. doi: 10.1111/biom.12682

Karlebach G, Shamir R (2008) Modelling and analysis of gene regulatory networks. Nature reviews. Molecular cell biology 9(10):770-780. doi: 10.1038/nrm2503

Karr JR, Sanghvi JC, Macklin DN, Gutschow MV, Jacobs JM, Bolival B, Assad-Garcia N, Glass JI, Covert MW (2012) A Whole-Cell Computational Model Predicts Phenotype from Genotype. Cell 150(2):389-401.

doi: 10.1016/i.cell.2012.05.044

Klepikova AV, Logacheva MD, Dmitriev SE, Penin AA (2015) RNA-seq analysis of an apical meristem time series reveals a critical point in Arabidopsis thaliana flower initiation. BMC Genomics 16:466. doi: 10.1186/s12864 015-1688-9

Rauber T, Rünger G (2012) Parallele Programmierung, 3. Aufl. 2013. eXamen.press. Springer, Berlin and Heidelberg. ISBN: 978-3-642-13603-0. doi: 10.1007/978-3-642$13604-7$

Reiß T (2002) Systembiologie: Systeme des Lebens. Bundesministerium für Bildung und Forschung

Zoppoli P, Morganella S, Ceccarelli M (2010) TimeDelayARACNE: Reverse engineering of gene networks from time-course data by an information theoretic approach. BMC Bioinformatics 11:154. doi: 10.1186/1471-2105-11154

\section{AUTOREN}

\section{Marco Schäfer}

Christian Jetschni

Dr. Chong Wang

Prof. Dr. Heike Pospisil

Professur für Life Science Informatics

Arbeitsgruppe "High Performance Computing

in Life Sciences"

Technische Hochschule Wildau

E-Mail für Korrespondenz:

heike.pospisil@th-wildau.de

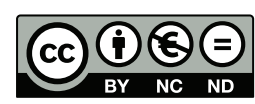

